UCRHEP-T492

June 2010

\title{
Quark and Lepton Flavor Triality
}

\author{
Ernest Ma \\ Department of Physics and Astronomy, University of California, \\ Riverside, California 92521, USA
}

\begin{abstract}
Motivated by the success of $A_{4}$ in explaining neutrino tribimaximal mixing, and its approximate residual $Z_{3}$ symmetry in the quark and charged-lepton sectors, the notion of flavor triality is proposed. Under this hypothesis, certain processes such as $\tau^{+} \rightarrow \mu^{+} \mu^{+} e^{-}$and $\tau^{+} \rightarrow e^{+} e^{+} \mu^{-}$are favored, but $\tau^{+} \rightarrow \mu^{+} e^{+} e^{-}$and $\mu^{+} \rightarrow e^{+} e^{+} e^{-}$ are disfavored. Similarly, $B^{0} \rightarrow \tau^{+} e^{-}$is favored, but $B^{0} \rightarrow \tau^{-} e^{+}$is disfavored.
\end{abstract}


Introduction : The observed neutrino mixing matrix is very close to the tribimaximal form [1] and is best understood in terms of the tetrahedral symmetry $A_{4}$ [2, 3, 4, [5]. The key to its success is the pattern of symmetry breaking with preserved subgroups [6, 17] such that $A_{4} \rightarrow Z_{3}$ in the charged-lepton sector and $A_{4} \rightarrow Z_{2}$ in the neutrino sector, which may be accomplished [8] in a renormalizable model using Higgs doublets and triplets, both transforming as $\underline{1}$ and $\underline{3}$ of $A_{4}$ alone [9]. Other more complicated scenarios with additional auxiliary symmetries have also been proposed [10, 11, 12, 13, 14, 15, 16, 17, 18, 19.

In the quark sector, the same pattern for both up and down quarks as the charged-lepton sector is the most natural choice, in which case there is perfect alignment of the two mass matrices, resulting in no mixing at all. Since quark mixing angles are known to be small, this is a good first approximation. Thus the residual symmetry $Z_{3}$ is approximately valid in all fermion sectors, except the neutrino sector. The notion of flavor triality in the quark and charged-lepton sectors is then a useful tool for understanding if $A_{4}$ (or some other symmetry which also breaks to $Z_{3}$ ) is the correct underlying explanation of neutrino tribimaximal mixing.

Flavor Triality: In most applications of the non-Abelian discrete flavor symmetry $A_{4}$ to quark and lepton mass matrices, $A_{4}$ is broken spontaneously and softly to the residual symmetry $Z_{3}$ in the up and down quark and charged-lepton sectors, and to $Z_{2}$ in the neutrino sector. The latter is actually a small perturbation, which is crucial for deriving the tribimaximal mixing of the neutrino mass matrix, but otherwise not very important in other physical processes. In fact, the Lagrangian is approximately invariant under $Z_{3}$ in such a scenario. Let $\omega=\exp (2 \pi i / 3)=-1 / 2+i \sqrt{3} / 2$ with $\omega^{3}=1$, then the quarks and charged leptons may be classified according to [8]

$$
\begin{aligned}
& u, d, e \sim 1, \quad c, s, \mu \sim \omega^{2}, \quad t, b, \tau \sim \omega \\
& u^{c}, d^{c}, e^{c} \sim 1, \quad c^{c}, s^{c}, \mu^{c} \sim \omega, \quad t^{c}, b^{c}, \tau^{c} \sim \omega^{2} .
\end{aligned}
$$


Therefore, certain quark and lepton flavor-violating processes are favored, but others are disfavored.

Using Eqs. (1) and (2), it is clear that only the following flavor-changing leptonic decays are favored:

$$
\tau^{+} \rightarrow \mu^{+} \mu^{+} e^{-}, \quad \tau^{+} \rightarrow e^{+} e^{+} \mu^{-} .
$$

In the quark sector, they are

$$
b \rightarrow s s \bar{d}, \quad b \rightarrow d d \bar{s}, \quad t \rightarrow c c \bar{u}, \quad t \rightarrow u u \bar{c} .
$$

In processes involving both quarks and leptons, they are

$$
\begin{aligned}
& B^{0} \rightarrow \tau^{+} e^{-}, \mu^{+} \tau^{-}, e^{+} \mu^{-}, \quad B_{s}^{0} \rightarrow \tau^{+} \mu^{-}, \mu^{+} e^{-}, e^{+} \tau^{-}, \\
& D^{0} \rightarrow \tau^{-} e^{+}, e^{-} \mu^{+}, \quad K^{0} \rightarrow \mu^{+} e^{-}, \quad \tau^{+} \rightarrow \mu^{+} K^{0}, \quad \tau^{+} \rightarrow e^{+} \bar{K}^{0} .
\end{aligned}
$$

Scalar Mediators: The mediators of flavor triality are a triplet (under $A_{4}$ ) of Higgs scalar doublets (under $S U(2)_{L} \times U(1)_{Y}$ ) which transform under $Z_{3}$ as

$$
\phi_{0} \sim 1, \quad \phi_{1} \sim \omega, \quad \phi_{2} \sim \omega^{2} .
$$

As for their Yukawa couplings to quarks and leptons, there are two existing versions. One [2, 18] comes from having $l_{1,2,3}^{c}$ transforming as $\underline{1}, \underline{1}^{\prime}, \underline{1}^{\prime \prime}$ under $A_{4}$. The other [8, 9] uses $\underline{3}$. In the former, $\phi_{0}$ is identified as the one Higgs doublet of the Standard Model, with couplings $\left(g / \sqrt{2} M_{W}\right)\left[m_{e} \bar{e}_{L} e_{R}+m_{\mu} \bar{\mu}_{L} \mu_{R}+m_{\tau} \bar{\tau}_{L} \tau_{R}\right]$ and similarly for quarks. The leptonic interactions of $\phi_{1,2}$ are given by [2]

$$
\begin{aligned}
\mathcal{L}_{\text {int }} & =\sqrt{\frac{3}{2}} \frac{g}{M_{W}}\left[m_{\tau}{\overline{\left(\nu_{\mu}, \mu\right)}}_{L} \tau_{R}+m_{\mu}{\overline{\left(\nu_{e}, e\right)}}_{L} \mu_{R}+m_{e}{\overline{\left(\nu_{\tau}, \tau\right)}}_{L} e_{R}\right] \phi_{1} \\
& +\sqrt{\frac{3}{2}} \frac{g}{M_{W}}\left[m_{\tau}{\overline{\left(\nu_{e}, e\right)}}_{L} \tau_{R}+m_{\mu}{\overline{\left(\nu_{\tau}, \tau\right)}}_{L} \mu_{R}+m_{e}{\overline{\left(\nu_{\mu}, \mu\right)}}_{L} e_{R}\right] \phi_{2}+\text { H.c. },
\end{aligned}
$$


whereas those involving quarks are

$$
\begin{aligned}
\mathcal{L}_{\text {int }} & =\sqrt{\frac{3}{2}} \frac{g}{M_{W}}\left[m_{b} \overline{(c, s)}_{L} b_{R}+m_{s} \overline{(u, d)}_{L} s_{R}+m_{d} \overline{(t, b)}_{L} d_{R}\right] \phi_{1} \\
& +\sqrt{\frac{3}{2}} \frac{g}{M_{W}}\left[m_{b} \overline{(u, d)}_{L} b_{R}+m_{s} \overline{(t, b)}_{L} s_{R}+m_{d} \overline{(c, s)}_{L} d_{R}\right] \phi_{2} \\
& +\sqrt{\frac{3}{2}} \frac{g}{M_{W}}\left[m_{t} \overline{(c, s)}_{L} t_{R}+m_{c} \overline{(u, d)}_{L} c_{R}+m_{u} \overline{(t, b)}_{L} u_{R}\right] \tilde{\phi}_{2} \\
& +\sqrt{\frac{3}{2}} \frac{g}{M_{W}}\left[m_{t} \overline{(u, d)}_{L} t_{R}+m_{c} \overline{(t, b)}_{L} c_{R}+m_{u} \overline{(c, s)}_{L} u_{R}\right] \tilde{\phi}_{1}+H . c .,
\end{aligned}
$$

where $\tilde{\phi}=i \sigma_{2} \phi^{*}$. Note that flavor-changing radiative decays such as $\mu \rightarrow e \gamma$ and $b \rightarrow s \gamma$ are not induced by these interactions.

The charged scalars $\phi_{1,2}^{ \pm}$are degenerate in mass [2]. However, the situation is more complicated [18] for the neutral scalars. Since $\phi_{1}^{0}, \bar{\phi}_{2}^{0}$ transform as $\omega$ under $Z_{3}$, whereas $\phi_{2}^{0}, \bar{\phi}_{1}^{0}$ transform as $\omega^{2}$, neither $\phi_{1}^{0}$ nor $\phi_{2}^{0}$ are mass eigenstates. Rather, they are

$$
\psi_{1,2}^{0}=\left(\phi_{1}^{0} \pm \bar{\phi}_{2}^{0}\right) / \sqrt{2} \sim \omega
$$

with $m_{1} \neq m_{2}$. Note also that in the Higgs potential itself, soft breaking of $A_{4}$ to $Z_{3}$ allows $\phi_{1,2}$ to have a mass different from that of $\phi_{0}$. This mass is not related to electroweak symmetry breaking and subject only to specific phenomenological constraints, as will be discussed.

In the latter version [8, 9, there is an additional Higgs doublet $\eta$ transforming as $\underline{1}$ under $A_{4}$. Whereas $\eta^{0}$ with $\left\langle\eta^{0}\right\rangle=v_{0}$ couples to charged leptons according to

$$
\frac{1}{3 v_{0}}\left(m_{e}+m_{\mu}+m_{\tau}\right)\left(\bar{e}_{L} e_{R}+\bar{\mu}_{L} \mu_{R}+\bar{\tau}_{L} \tau_{R}\right)
$$

$\phi_{0}$ of Eq. (7) with $\left\langle\phi_{0}^{0}\right\rangle=v$ couples according to

$$
\frac{1}{3 v}\left[\left(2 m_{e}-m_{\mu}-m_{\tau}\right) \bar{e}_{L} e_{R}+\left(2 m_{\mu}-m_{\tau}-m_{e}\right) \bar{\mu}_{L} \mu_{R}+\left(2 m_{\tau}-m_{e}-m_{\mu}\right) \bar{\tau}_{L} \tau_{R}\right] .
$$

Hence the linear combination $\left(v_{0} \eta+v \phi_{0}\right) / \sqrt{v_{0}^{2}+v^{2}}$ acts as the Standard-Model Higgs doublet, and the orthogonal combination has enhanced couplings [20] to $\bar{e}_{L} e_{R}$ and $\bar{\mu}_{L} \mu_{R}$. 
The Yukawa couplings of $\phi_{1,2}^{0}$ to leptons are now given by

$$
\begin{aligned}
& \frac{\phi_{1}^{0}}{3 v}\left[\left(2 m_{\tau}-m_{e}-m_{\mu}\right) \bar{e}_{L} \mu_{R}+\left(2 m_{e}-m_{\mu}-m_{\tau}\right) \bar{\mu}_{L} \tau_{R}+\left(2 m_{\mu}-m_{\tau}-m_{e}\right) \bar{\tau}_{L} e_{R}\right]+ \\
& \frac{\phi_{2}^{0}}{3 v}\left[\left(2 m_{\mu}-m_{\tau}-m_{e}\right) \bar{e}_{L} \tau_{R}+\left(2 m_{\tau}-m_{e}-m_{\mu}\right) \bar{\mu}_{L} e_{R}+\left(2 m_{e}-m_{\mu}-m_{\tau}\right) \bar{\tau}_{L} \mu_{R}\right]+H . c .
\end{aligned}
$$

and those to quarks are

$$
\begin{aligned}
& \frac{\phi_{1}^{0}}{3 v}\left[\left(2 m_{b}-m_{d}-m_{s}\right) \bar{d}_{L} s_{R}+\left(2 m_{d}-m_{s}-m_{b}\right) \bar{s}_{L} b_{R}+\left(2 m_{s}-m_{b}-m_{d}\right) \bar{b}_{L} d_{R}\right]+ \\
& \frac{\phi_{2}^{0}}{3 v}\left[\left(2 m_{s}-m_{b}-m_{d}\right) \bar{d}_{L} b_{R}+\left(2 m_{b}-m_{d}-m_{s}\right) \bar{s}_{L} d_{R}+\left(2 m_{d}-m_{s}-m_{b}\right) \bar{b}_{L} s_{R}\right]+ \\
& \frac{\bar{\phi}_{2}^{0}}{3 v}\left[\left(2 m_{t}-m_{u}-m_{c}\right) \bar{u}_{L} c_{R}+\left(2 m_{u}-m_{c}-m_{t}\right) \bar{c}_{L} t_{R}+\left(2 m_{c}-m_{t}-m_{u}\right) \bar{t}_{L} u_{R}\right]+ \\
& \frac{\bar{\phi}_{1}^{0}}{3 v}\left[\left(2 m_{c}-m_{t}-m_{u}\right) \bar{u}_{L} t_{R}+\left(2 m_{t}-m_{u}-m_{c}\right) \bar{c}_{L} u_{R}+\left(2 m_{u}-m_{c}-m_{t}\right) \bar{t}_{L} c_{R}\right]+\text { H.c. }
\end{aligned}
$$

Contrary to Eqs. (8) and (9), where many of the Yukawa couplings are proportional to the masses of light quarks and leptons, all the couplings in Eqs. (13) and (14) are proportional to the largest mass in each sector, i.e. $m_{\tau}, m_{b}$, and $m_{t}$. From the nonobservation of the rare decays listed in Eqs. (3) to (6), this would require $m_{1,2}$ to be much greater than the constraints coming from Eqs. (8) and (9).

Phenomenological Constraints : The best experimental limit on the rare decays listed in Eqs. (3) to (6) comes from $K_{L}^{0} \rightarrow \mu^{ \pm} e^{\mp}$ with a branching fraction [21] less than $4.7 \times 10^{-12}$. Using Eqs. (8) and (9), with $m_{K}=495 \mathrm{MeV}, m_{s}=104 \mathrm{MeV}, V_{u s}=0.225$, the ratio

$$
\frac{\Gamma\left(K_{L}^{0} \rightarrow \mu^{ \pm} e^{\mp}\right)}{\Gamma\left(K^{+} \rightarrow \mu^{+} \nu\right)}=\frac{9 m_{K}^{2} m_{s}^{2}}{4\left|V_{u s}\right|^{2}}\left(\frac{1}{m_{1}^{2}}+\frac{1}{m_{2}^{2}}\right)^{2}
$$

leads to the bound

$$
\frac{m_{1} m_{2}}{\sqrt{m_{1}^{2}+m_{2}^{2}}}>510 \mathrm{GeV}
$$


Using the above bound, the branching fraction of the rare decay $B^{0} \rightarrow \tau^{+} e^{-}$is then predicted by Eqs. (8) and (9) to be less than $1.4 \times 10^{-7}$, well below the current experimental bound of $1.1 \times 10^{-4}$. The other flavor-changing leptonic decays of $B^{0}$ (i.e. $\mu^{+} \tau^{-}$and $e^{+} \mu^{-}$) are suppressed even further, i.e. by $m_{\mu}^{2} / m_{\tau}^{2}$ and $m_{e}^{2} / m_{\tau}^{2}$ respectively. Similarly, the branching fraction of $B_{s}^{0} \rightarrow \tau^{+} \mu^{-}$is predicted to be roughly equal to that of $B^{0} \rightarrow \tau^{+} e^{-}$, and the other modes $\mu^{+} e^{-}$and $e^{+} \tau^{-}$are suppressed analogously. The decay rates of $\tau^{+} \rightarrow \mu^{+} \mu^{+} e^{-}$and $\tau^{+} \rightarrow e^{+} e^{+} \mu^{-}$are proportional to $m_{\mu}^{2} m_{\tau}^{2}\left(m_{1}^{2}+m_{2}^{2}\right)^{2} / m_{1}^{4} m_{2}^{4}$ and $m_{e}^{2} m_{\tau}^{2}\left(m_{1}^{2}+m_{2}^{2}\right)^{2} / m_{1}^{4} m_{2}^{4}$ respectively and thus many orders of magnitude below current limits. Similarly, the decay rates of $D^{0} \rightarrow \tau^{-} e^{+}$and $D^{0} \rightarrow e^{-} \mu^{+}$are proportional to $m_{\tau}^{2} m_{c}^{2}\left(m_{1}^{2}+m_{2}^{2}\right)^{2} / m_{1}^{4} m_{2}^{4}$ and $m_{e}^{2} m_{c}^{2}\left(m_{1}^{2}+m_{2}^{2}\right)^{2} / m_{1}^{4} m_{2}^{4}$ respectively, and are also negligible.

Higgs Decays : If the neutral Higgs bosons $\psi_{1,2}^{0}$ of Eq. (10) are observed. Their decays are then completely determined according to Eq. (8) and (9). They should decay dominantly into $t \bar{c}$ and $t \bar{u}$.

In the version with Eqs. (11) to (14), $\phi_{1,2}$ are presumably too heavy to be observed. However, $\eta$ and $\phi_{0}$ may be light, and their decays according to Eqs. (11) and (12) would also be indicative of flavor triality.

Lepton Flavor Triality Alone : The notion of flavor triality is much more applicable to charged leptons than quarks, because neutrinos are nearly massless. If quarks are not considered, then only the decays of Eq. (3) are relevant and only Eqs. (8) and (13) are to be studied. Using Eq. (8),

$$
B\left(\tau^{+} \rightarrow \mu^{+} \mu^{+} e^{-}\right)=\frac{9 m_{\tau}^{2} m_{\mu}^{2}\left(m_{1}^{2}+m_{2}^{2}\right)^{2}}{m_{1}^{4} m_{2}^{4}} B(\tau \rightarrow \mu \nu \nu)<2.3 \times 10^{-8},
$$

the bound

$$
\frac{m_{1} m_{2}}{\sqrt{m_{1}^{2}+m_{2}^{2}}}>39 \mathrm{GeV}
$$

is obtained instead of Eq. (16). The branching fraction $B\left(\tau^{+} \rightarrow e^{+} e^{+} \mu^{-}\right)$is further sup- 
pressed by $m_{e}^{2} / m_{\mu}^{2}$ in this case. On the other hand, the two branching fractions are about the same if Eq. (13) is used, yielding a bound of $54 \mathrm{GeV}$ instead. This means that $\psi_{1,2}^{0}$ may be light enough to be accessible at the Large Hadron Collider (LHC). Their production [22] is presumably via $Z \rightarrow \psi_{1,2}^{0} \bar{\psi}_{1,2}^{0}$. Using Eq. (8), $\psi_{1,2}^{0}$ are predicted to decay equally into $\tau^{+} \mu^{-}$ and $\tau^{-} e^{+}$, whereas the $\mu^{+} e^{-}$mode is suppressed by $m_{\mu}^{2} / m_{\tau}^{2}$. Using Eq. (13), the decay rates of $\psi_{1,2}^{0}$ into $\tau^{+} \mu^{-}$and $\tau^{-} e^{+}$are again roughly equal, but that to $\mu^{+} e^{-}$is now about four times larger.

Conclusion : The notion of flavor triality for quarks and leptons may a useful guide for checking if $Z_{3}$ is an underlying residual symmetry for understanding neutrino tribimaximal mixing from either $A_{4}$ or some other non-Abelian diecrete symmetry which also breaks to $Z_{3}$. In two specific scenarios with scalar mediators, flavor-changing quark and lepton interactions are discussed. If only lepton flavor triality is valid, these scalars may be light enough to be observable at the LHC and their decays would reveal their underlying flavor structure.

Acknowledgement: This work was supported in part by the U. S. Department of Energy under Grant No. DE-FG03-94ER40837.

\section{References}

[1] P. F. Harrison, D. H. Perkins, and W. G. Scott, Phys. Lett. B530, 167 (2002).

[2] E. Ma and G. Rajasekaran, Phys. Rev. D64, 113012 (2001).

[3] K. S. Babu, E. Ma, and J. W. F. Valle, Phys. Lett. 552, 207 (2003).

[4] E. Ma, Phys. Rev. D70, 031901 (2004).

[5] G. Altarelli and F. Feruglio, Nucl. Phys. B720, 64 (2005).

[6] C. S. Lam, Phys. Lett. B656, 193 (2007).

[7] A. Blum, C. Hagedorn, and M. Lindner, Phys. Rev. D77, 076004 (2008). 
[8] E. Ma, arXiv:0908.3165 [hep-ph] (Mod. Phys. Lett. A25, in press).

[9] E. Ma, Mod. Phys. Lett. A21, 2931 (2006).

[10] K. S. Babu and X.-G. He, arXiv:0507217 [hep-ph].

[11] G. Altarelli and F. Feruglio, Nucl. Phys. B741, 215 (2006).

[12] E. Ma, Phys. Rev. D73, 057304 (2006).

[13] X.-G. He, Nucl. Phys. Proc. Suppl. 168, 350 (2007).

[14] S. Morisi, M. Picariello, and E. Torrente-Lujan, Phys. Rev. D75, 075015 (2007).

[15] E. Ma, Mod. Phys. Lett. A22, 101 (2007).

[16] G. Altarelli, F. Feruglio, and C. Hagedorn, JHEP 0803, 052 (2008).

[17] F. Bazzochi, M. Frigerio, and S. Morisi, Phys. Rev. D78, 116018 (2008).

[18] E. Ma, Phys. Lett. B671, 366 (2009).

[19] G. Altarelli and D. Meloni, J. Phys. G36, 085005 (2009).

[20] C. S. Lam, Phys. Lett. B689, 169 (2010).

[21] Particle Data Group, C. Amsler et al., Phys. Lett. B667, 1 (2008).

[22] Q.-H. Cao, E. Ma, and G. Rajasekaran, Phys. Rev. D76, 095011 (2007). 